OPEN ACCESS

Edited by:

Xiangfeng Kong,

Institute of Subtropical Agriculture,

CAS, China

Reviewed by:

Bi E. Tan,

Hunan Agricultural University, China

Jinming You,

Jiangxi Agricultural University, China

${ }^{*}$ Correspondence:

Zhiru Tang

tangzhiru2326@sina.com

Specialty section:

This article was submitted to

Food Microbiology,

a section of the journal

Frontiers in Microbiology

Received: 20 October 2021 Accepted: 13 December 2021

Published: 22 February 2022

Citation:

Mohamed TM, Sun W,

Bumbie GZ, Elokil $A A$,

Mohammed KAF, Zebin R, Hu P, Wu L and Tang $Z$ (2022) Feeding Bacillus subtilis ATCC19659 to Broiler

Chickens Enhances Growth Performance and Immune Function by Modulating Intestinal Morphology

and Cecum Microbiota.

Front. Microbiol. 12:798350. doi: 10.3389/fmicb.2021.798350

\section{Feeding Bacillus subtilis ATCC19659 to Broiler Chickens Enhances Growth Performance and Immune Function by Modulating Intestinal Morphology and Cecum Microbiota}

Taha M. Mohamed ${ }^{1,2}$, Weizhong Sun', Gifty Z. Bumbie', Abdelmotaleb A. Elokil' ${ }^{3}$, Khaled Abuelezz Fouad Mohammed ${ }^{4}$, Rao Zebin ${ }^{1}$, Ping $\mathrm{Hu}^{1}$, Liuting $\mathrm{Wu}^{1}$ and Zhiru Tang ${ }^{1 *}$

\footnotetext{
' Laboratory for Bio-Feed and Molecular Nutrition, College of Animal Science and Technology, Southwest University, Chongqing, China, ${ }^{2}$ Department of Animal and Fish Production, Faculty of Agriculture (Saba Basha), Alexandria University, Alexandria, Egypt, ${ }^{3}$ Animal Production Department, Faculty of Agriculture, Benha University, Moshtohor, Egypt,

${ }^{4}$ Department of Poultry Production, Faculty of Agriculture, Assiut University, Assiut, Egypt
}

This study investigated dietary supplementation with Bacillus subtilis (BS) ATCC19659 on growth performance, biochemical indices, intestinal morphology, and cecum microflora in broiler chicks. A total of 600 Arbor 1-day Acres broilers of either sex were allotted to 5 treatments: chicks were fed a corn- and soybean-based diet (CON); chicks were fed basal diet containing $500 \mathrm{mg} \mathrm{ZnB/kg} \mathrm{(ZnB);} \mathrm{chicks} \mathrm{were} \mathrm{fed} \mathrm{basal}$ diet containing $1 \times 10^{8} \mathrm{CFU} / \mathrm{g}$ feed of BS-ATCC19659 (BS-1); chicks were fed basal diet containing $3 \times 10^{8} \mathrm{CFU} / \mathrm{g}$ feed of BS-ATCC19659 (BS-3); and chicks were fed basal diet containing $5 \times 10^{8} \mathrm{CFU} / \mathrm{g}$ feed of BS-ATCC19659 (BS-5). Each treatment comprised 6 replicates with 20 birds for each replicate pen. Chicks in the BS-5 and BS-3 groups had higher body weight at the $21^{\text {st }}$ and $42^{\text {nd }}$ days and average daily gain from 1 to 21 days than that in the CON group $(p<0.05)$. Chicks in the BS- 5 and ZnB groups had higher serum antioxidant activities and immunity response than those in the CON group $(p<0.05)$. Compared with the CON group, the liver mRNA abundance of GHR, TGF- $\beta, I G F-1$, IFN- $\gamma$, SOD, CAT, and GPX of chicks in three BS groups and the ileum villus length $(\mu \mathrm{m})$ of chicks in BS-3 and ZnB groups was increased $(p<0.05)$. Compared with the CON group, the villus height-to-crypt depth ratio of the ileum of chicks in the BS-5 and BS-3 groups and the crypt depth and villus height-to-crypt depth ratio of the jejunum in the BS- 5 and ZnB groups were increased $(p<0.05)$. The abundance of the Cyanobacteria phyla in the cecum decreased in response to treatment with both BS-ATCC19659 and ZnB groups ( $p<0.05)$. Compared with the CON group, the cecum abundance of genera GCA-900066575 (Lachnospiraceae), Anaerofustis, and Papillibacter (Firmicutes phylum) in three BS groups were increased $(p<0.05$ ); 
The abundance of genus Escherichia-Shigella reduced in the BS-3 group $(p<0.05)$. Compared with the CON group, the cecum abundance of genus Clostridia_unclassified in ZnB and BS-5 groups was decreased $(p<0.05)$ of broilers. Generally, Bacillus subtilis ATCC19659 as feed additive positively affected growth performance, immunity response, and cecal microflora of broilers.

Keywords: growth performance, immune response, intestinal morphology, cecum microbiota, broiler, Bacillus subtilis ATCC19659

\section{INTRODUCTION}

Antibiotics were used to eliminate poultry pathogens and promote growth performance and improve egg production. However, because of the potential of antibiotic-resistant strains of pathogenic organisms spreading into the environment, human infection through the food chain can lead to serious consequences to public health (Singer et al., 2003). Limiting antibiotics as growth enhancers in livestock production prompted a need to evaluate and present new alternatives to growth promoters (Tang et al., 2017). Indeed, this is primarily to avoid pathogenic bacterial resistance to antibiotics and to meet the growing consumer consciousness about hazardous residues in poultry meat and eggs. On the other hand, banning antibiotics in the poultry industry would result in reduced profits due to reduced flock productivity (Yang et al., 2019). Therefore, some viable alternatives to dietary antibiotics, such as probiotics, prebiotics, and organic acids, have been suggested in this regard to promote healthy flock productivity (Diaz-Sanchez et al., 2015; Suresh et al., 2018). Compared to antibiotics, these alternatives were characterized by natural environmental safety, no harmful residues accumulating in poultry eggs and meat, promoting a healthy gut microbiota and improving feed intake, feed conversion, and growth rate (Gadde et al., 2017).

Zinc bacitracin $(\mathrm{ZnB})$ is an antibiotic commonly used in dietary commercial broiler production. Zinc bacitracin is a combination of high molecular weight polypeptides produced by Bacillus licheniformis, considered a growth promoter (Pedroso et al., 2006; Crisol-Martínez et al., 2017). Bacillus subtilis is commonly included in probiotic supplement formulations as a healthy probiotic strain to improve growth performance and enhance immune and digestive system health (Bai et al., 2017). It consumes a high amount of free oxygen during colonization in the gut, restricting the growth of pathogenic aerobic bacteria to enhance the growth of anaerobic bacteria such as Lactobacillus and Bifidobacterium (Gao et al., 2017). Previous studies demonstrated that dietary supplementation of Bacillus subtilis improved growth performance (Amerah et al., 2013; Jayaraman et al., 2017; Wang et al., 2017), enhanced immune functions (Rajput et al., 2013; Lee et al., 2014; Bai et al., 2017; Liu et al., 2019), increased the absorption of nutrients through a positive influence on intestinal morphology (Frizzas de et al., 2003; Palamidi et al., 2016; Zhang et al., 2016; He et al., 2019), improved antioxidant capacity (He et al., 2019; Liu et al., 2019), and affected the composition of the broiler microflora (Hong et al., 2019; Li et al., 2019). Lee et al. (2014) reported that supplementing dietary Bacillus subtilis in pigs significantly affected microbiota compositions and improved immune function.

Gut microbiota (GM) is the totality of microorganisms of bacteria, viruses, protozoa, and fungi that have colonized the gastrointestinal tract of the host. It regulates energy harvest from diet, intestinal immunity, and metabolic performance to identify the host productivity (Elokil et al., 2020a). GM could be modified by numerous techniques, including administration of synbiotics, probiotics, antibiotics, prebiotics, and fecal transplantation (Cammarota et al., 2014; Elokil et al., 2020b).

This present study was conducted to investigate Bacillus subtilis ATCC19659 (BS-ATCC19659) as a substitute for antibiotics in poultry diets. Therefore, this study aimed to elucidate host-microbiome interactions under the influence of dietary supplementation at three different concentrations of BS-ATCC19659 on the host performance of broiler (growth, antioxidant status, immunity, and gastrointestinal responses) and the intestinal microbial community.

\section{MATERIALS AND METHODS}

\section{Ethics Statement}

Experimental procedures were approved by the License of Experimental Animals (SYXK 2014-0002) of the Animal Experimentation Ethics Committee of Southwest University, Chongqing, China, and birds were raised following the guidelines described by the Animal Care Committee of Chongqing, China. In addition, efforts were made to reduce animal suffering and were carried out in compliance with the "ARRIVE" guidelines for reporting in vivo experiments in animal research.

\section{Bacterial Strain}

Bacillus subtilis ATCC19659 (BS-ATCC19659) was provided from (KWIk-STIk ${ }^{\mathrm{TM}}$, Microbiologics, Microbiologics, Inc., Saint Cloud, United States), and total content from Bacillus subtilis was prepared of a viable bacterium by serial dilution plate counts after culturing in nutrient agar at $37^{\circ} \mathrm{C}$ for $14 \mathrm{~h}$ under an aerobic environment.

\section{Experimental Design and Management}

Six hundred day-of-hatch Arbor Acres broiler chicks were individually weighed and randomly assigned to five groups with six replicates per group and 20 chicks per replicate. The broiler chickens were fed a corn-soybean-based diet. Birds in the control group (CON group) were fed a basal diet without BS-ATCC19659 
and antibiotic, those in the $\mathrm{ZnB}$ group were fed a basal diet with $500 \mathrm{mg} / \mathrm{kg}$ of zinc bacitracin, and BS-ATCC19659 groups were fed a basal diet with Bacillus subtilis ATCC19659 at $1 \times 10^{8}$ $\mathrm{CFU} / \mathrm{g}$ feed (BS-1 group), $3 \times 10^{8} \mathrm{CFU} / \mathrm{g}$ feed (BS-3 group), and $5 \times 10^{8} \mathrm{CFU} / \mathrm{g}$ feed (BS-5 group). Mashed feed and water were provided ad libitum, and a constant lighting program was used. The basal diet was formulated to meet NRC (1994) requirements during the starter (1-21 days) and finisher periods (22-42 days). The composition and the calculated nutrient content of the experimental diet are shown in Table 1. Every morning, before the inclusion of BS-ATCC19659, all the birds had their water and feed withdrawn for about $3 \mathrm{~h}$. After this period, the feed was mixed with (BS-ATCC19659) with different concentrations, and birds consumed them within $30 \mathrm{~min}$. Moreover, birds in $\mathrm{CON}$ and $\mathrm{ZnB}$ groups consumed a similar amount of their usual feed. Once feed was finished, the regular water and feed were restored to birds. The adding of BS-ATCC19659 in feed was in final concentrations of 1,3 , and $5 \times 10^{8} \mathrm{CFU} / \mathrm{g}$, as described by Bortoluzzi et al. (2019). All birds were housed in cages of the same size $(30 \times 175 \times 155 \mathrm{~cm})$ at $33^{\circ} \mathrm{C}$ and reduced by $3^{\circ} \mathrm{C}$ each week to $24^{\circ} \mathrm{C}$ until the end of the experiment. At the age of 2 weeks, chicks in all groups were vaccinated against essential Newcastle disease $\left(\mathrm{YEBIO}^{\circledR}\right.$, Qingdao, China) using the LaSota B1 Strain of Newcastle disease virus in live freeze-dried form by adding to their drinking water.

TABLE 1 | Ingredients and nutrients composition of the basal diets (DM basis).

\begin{tabular}{lcc}
\hline Ingredients (\%) & Starter (1-21 days) & Finisher (21-42 days) \\
\hline Corn & 58.00 & 63.00 \\
Soybean $(44 \% \mathrm{CP})^{\mathrm{a}}$ & 28.20 & 24.90 \\
Gluten $(60 \% \mathrm{CP})^{\mathrm{b}}$ & 7.00 & 6.00 \\
Dicalcium phosphate & 1.80 & 1.20 \\
Limestone & 1.30 & 1.30 \\
Soybean oil & 2.00 & 2.00 \\
Salt & 0.30 & 0.30 \\
L-Lysine HCl & 0.20 & 0.20 \\
DL-Methionine & 0.20 & 0.10 \\
Premix & 1.00 & 1.00 \\
Total & 100 & 100
\end{tabular}

\begin{tabular}{lcc}
\hline Calculated composition & & \\
$\mathrm{ME}(\mathrm{kcal} / \mathrm{kg})^{\mathrm{d}}$ & $3,012.26$ & $3,068.97$ \\
Crude protein (\%) $_{\text {Calcium (\%) }}$ & 21.99 & 20.28 \\
Total phosphor (\%) & 0.98 & 0.84 \\
Methionine (\%) & 0.72 & 0.59 \\
Methionine + cysteine (\%) & 0.59 & 0.47 \\
Lysine (\%) & 0.95 & 0.80 \\
\hline
\end{tabular}

a,b Crude protein.

${ }^{c}$ Provided the following per kilogram of diet: $13,000 \mathrm{IU}$ of vitamin A; 1,300 IU of vitamin D; $65 \mathrm{U}$ of vitamin E; $3.4 \mathrm{mg}$ of menadione; $37 \mathrm{mg}$ of pantothenic acid; $6.6 \mathrm{mg}$ of riboflavin; $3.7 \mathrm{mg}$ of folic acid; $39 \mathrm{mg}$ of niacin; $1.0 \mathrm{mg}$ of thiamine; $4.3 \mathrm{mg}$ of vitamin B6; $0.23 \mathrm{mg}$ biotin; $0.075 \mathrm{mg}$ of vitamin B12: $43 \mathrm{mg}$ of choline chloride.

$170 \mathrm{mg}$ of zinc; $140 \mathrm{mg}$ of iron; $34 \mathrm{mg}$ of manganese; $16 \mathrm{mg}$ of copper; $0.29 \mathrm{mg}$ of iodine; $0.29 \mathrm{mg}$ of selenium. ${ }^{d}$ Metabolizable energy.

\section{Growth Performance}

Feed intake of chicks and the number of dead birds were recorded daily. Chicks were weighed on days 0,21 , and 42 before feeding in the morning. The average daily feed intake (ADFI), average daily weight gain (ADG), and feed conversion rate (FCR) were calculated based on the following formulas: ADFI $(\mathrm{g} / \mathrm{d})=$ total food intake (g)/feed days (d); ADG (g/d) = (final weight - initial weight) (g)/feed days (d); FCR (g/g) = ADFI (g/d)/ADG (g/d). Survivability rates $(\%)=($ Number of surviving birds at the end of experimental period/initial number of birds) $\times 100$.

\section{Measurements and Sampling}

At the end of the experimental period (age 42 days), the six birds with bodyweight close to the average bodyweight from each treatment were selected. Blood samples were collected from the jugular vein in non-heparinized tubes $(10.0 \mathrm{ml})$. The collected blood samples were centrifuged at $3,500 \mathrm{rpm}$ for $10 \mathrm{~min}$ at $4^{\circ} \mathrm{C}$ to harvest the serum. The serum was stored at $-20^{\circ} \mathrm{C}$ for biochemical analysis and ELISA. After blood sampling, the six birds selected from each treatment were anesthetized with an intravenous injection of sodium pentobarbital $(50 \mathrm{mg} / \mathrm{kg}$ BW) and exsanguinated, and segments of the mid-ileum and jejunum were collected, fixed with formalin for $48 \mathrm{~h}$ and paraffinembedded. A total of five samples from each treatment from both liver and jejunum tissue were immediately collected after slaughtering each group and stored at $-80^{\circ} \mathrm{C}$ until RNA isolation. A total of six samples from each treatment from both ceca were taken out after slaughtering from each group. Then, the cecal digesta samples were immediately collected in a sterilizing tube, frozen in liquid $\mathrm{N}_{2}$, and stored at $-80^{\circ} \mathrm{C}$ until further analysis.

\section{Serum-Biochemical Indexes}

The contents of serum malondialdehyde (MDA) and the activities of glutathione (GSH), total antioxidant capacity (T-SOD), catalase (CAT), and superoxide dismutase (SOD) were measured. Samples were analyzed by an autoanalyzer (SHIMADZU CL-8,000 automatic autoanalyzer, Shanghai, China), and commercial kits acquired from Nanjing Jiancheng Bioengineering Institute, Nanjing, China (NJJCBIO), ${ }^{1}$ were used. The serum immunoglobulin $\mathrm{M}$ ( $\operatorname{IgM})$, immunoglobulin $\mathrm{G}(\operatorname{IgG})$, immunoglobulin A ( $\operatorname{Ig} \mathrm{A})$, immunoglobulin $\mathrm{E}(\mathrm{IgE})$, secretory IgA (sIgA), complement component 3 (C3), and complement component 4 (C4) were determined using commercial kits by following the manufacturer's instructions (NJJCBIO). In addition, interleukins IL-4, IL-6, IL-10, tumor necrosis factor- $\alpha$ (TNF- $\alpha$ ), and transforming growth factor-beta (TGF- $\beta$ ) in serum were measured by chicken-specific ELISA kits (NJJCBIO) following the manufacturer's instructions. The information of all kits is shown in Supplementary Table 1.

\section{H\&E Staining}

The jejunum and ileum morphology was analyzed based on the H\&E staining method described by Wang et al. (2009). From each sample, two sections $(100 \mu \mathrm{m})$ were obtained and

\footnotetext{
${ }^{1}$ http://www.njjcbio.com/
} 
stained with hematoxylin for $1 \mathrm{~min}$. After that, all sections were counterstained with eosin for $10 \mathrm{~s}$. All these variables were measured with a camera (Olympus; TH4-200; Tokyo, Japan) coupled with computer-assisted digital Image-Pro Plus (IPP) analysis software (Image-Pro Plus 4.5, Media Cybernetics, Silver Spring, MD, United States) to assess the maximum villus length, crypt depth, and submucosa/muscularis/serosa.

\section{Real-Time RT-PCR}

Total RNA was independently extracted from liver and jejunum samples $(n=5)$, using the TRIzol reagent (15596026, Ambion, Chongqing, China), according to the instructions of the manufacturer. Then, RNA was purified with DNase I (TaKaRa, Shiga, Japan) and an RNA clean kit (Tiangen, China). The quality and quantity of RNA were detected using a NanoDrop spectrophotometer (Thermo Scientific, Shanghai, China) at an absorption rate of 260:280 $\mathrm{nm}$ and using gel electrophoresis. To synthesize cDNA, total RNA was reverse-transcribed with MMLV reverse transcriptase (M1705, Promega, Chongqing, China), 5ÕM-MLV Buffer (M1705, Promega, Chongqing, China), and RNasin inhibitor (N2115, Promega, Chongqing, China), dNTPs (4030Q, Takara, Chongqing, China).

Quantitative real-time PCR (qRT-PCR) was done to determine the expression of seven target genes of growth hormone receptor $(G H R)$, transforming growth factor-beta (TGF- $\beta$ ), insulin-like growth factor-1 (IGF-1), interferon- $\gamma(I F N-\gamma)$, superoxide dismutase $(S O D)$, catalase $(C A T)$ and glutathione peroxidase $(G P X)$ using the qTOWER 3G Real-Time PCR System (Lead Scientific Technology, Chengdu, China) with the ChamQ Universal SYBR qPCR Master Mix (Q711-02, Vazyme, Chongqing, China) and performed in a total volume of $20 \mu \mathrm{l}$. A separation curve analysis step of 5 tissue samples from each group was amplified in triplicate. The relative gene expression mRNA was determined to those of the housekeeping gene for eukaryotic $\left(\beta\right.$-actin). The $2^{-\Delta \Delta \mathrm{Ct}}$ method was used to measure mRNA abundance for calculating the $\Delta \mathrm{Ct}$ value as $\left(\mathrm{Ct}_{\text {target }}-\mathrm{Ct}_{\mathrm{B}-\text { actin }}\right)$ treatment- $\left(\mathrm{Ct}_{\text {target }}-\mathrm{Ct}_{\mathrm{B}-\text { actin }}\right)$ control. The primer information for targeting genes is shown in Supplementary Table 2.

\section{Analysis of 16S rDNA Gene Sequencing}

For DNA extraction, cecum content samples collected on day 42 were beaded with a Mini-BeadBeater, and then the DNA was extracted using the Power Fecal DNA Isolation Kit (MO BIO, Carlsbad, CA, United States). DNA was stained using the Quant-iT Pico Green dsDNA Kit (Invitrogen Ltd., Paisley, United Kingdom) and quantified using a NanoDrop spectrophotometer (Nyxor Biotech, Paris, France).

For DNA MiSeq sequencing, PCR amplification of the $\mathrm{V} 4$ region of bacterial $16 \mathrm{~S}$ rDNA was performed using the universal primers 515F (5'-CCTACGGGNGGCWGCAG-3') and 806R (5'-GGACTACHVGGGTWTCTAAT-3'), incorporating the FLX Titanium adapters and a sample barcode sequence. The cycling parameters were as follows: $4 \mathrm{~min}$ of initial denaturation at $94^{\circ} \mathrm{C} ; 25$ cycles of denaturation at $94^{\circ} \mathrm{C}(30 \mathrm{~s})$, annealing at $50^{\circ} \mathrm{C}(45 \mathrm{~s})$, and elongation at $72^{\circ} \mathrm{C}(30 \mathrm{~s})$; and final extension at $72^{\circ} \mathrm{C}$ for $5 \mathrm{~min}$. Three separate PCR reactions for each sample were pooled for MiSeq sequencing. The PCR products were separated by $1.5 \%$ agarose gel electrophoresis and purified using the QIAquick Gel Extraction Kit (Qiagen, Hilden, Germany). Amplicons were quantified using the Quant-iT Pico Green dsDNA Assay Kit (Invitrogen, Carlsbad, CA, United States). Equal concentrations of amplicons were pooled from each sample. Libraries were constructed using the TruSeq DNA PCR-Free Sample Prep Kit, and MiSeq sequencing was performed with the MiSeq Reagent Kit v2 (Illumina, San Diego, CA, United States).

Based on unique barcodes of samples, paired-end reads were assigned to them and shortened by cutting off the barcode and primer sequence. Merging of paired-end reads was done using FLASH. The resulting sequences were further screened and filtered for quality and length. Under specific conditions of filtering, quality filtering on the raw reads was performed to obtain clean tags that are high in quality according to fqtrim (v0.94). V-search software (v2.3.4) was used in filtering chimeric sequences. Feature table and feature sequence were obtained after dereplication using DADA2. The relative abundance of each sample was normalized using feature abundance; this was done according to the SILVA (release 132) classifier. QIIME2 calculated alpha and beta diversities analysis; the graphs were drawn by the $\mathrm{R}$ package.

\section{Statistical Analysis}

Beta diversity analyses for the principal component analysis (PCA), principal coordinate analysis (PCoA), and nonmetric multidimensional scaling (NMDS) were used to obtain the comparative analysis of intergroup and group differences in terms of unique fraction distance. Alpha diversity analyses through five indices of chao1, observed OUT, Goods coverage, Shannon, and Simpson were analyzed by one-way ANOVA analysis, and the differences were compared using Duncan's test $(p<0.05)$. The permutational analysis of variance (PERMANOVA) analyzed the significant differences in beta diversity. The relative abundances at the phylum and genus were estimated by the Kruskal-Wallis test $(p<0.05)$. Data on growth performance, serum-biochemical indices, intestinal morphology, and gene expression in the liver and jejunum were analyzed using one-way ANOVA and performed using the general linear model (GLM) procedure of SAS (SAS, 2002, Cary, NC, United States, version 9). Individual chicks were considered as experimental units, and one fixed effect was included in the statistical model. All differences were considered significantly different at $p<0.05$. Pairwise comparisons were performed using Duncan's multiplerange test.

\section{RESULTS}

\section{Growth Performance Assessment of Host Broiler}

The growth performance results are shown in Table 2. An increase in weight was observed at the $21^{\text {st }}$ and $42^{\text {nd }}$ days of broiler in response to dietary BS-3 and BS-5 groups compared to the $\mathrm{CON}$ and $\mathrm{ZnB}$ groups $(p<0.05)$. Compared to chicks in the $\mathrm{ZnB}$ group, BS-5 and BS-3 groups display a significant 
TABLE 2 | Effect of dietary supplementation of Bacillus subtilis ATCC19659 on the broiler's growth performance.

\begin{tabular}{|c|c|c|c|c|c|c|c|}
\hline \multirow[t]{2}{*}{ Items $^{1}$} & \multicolumn{5}{|c|}{ Treatments $^{2}$} & \multirow[t]{2}{*}{ SEM } & \multirow[t]{2}{*}{$p$-value } \\
\hline & CON & $\mathrm{ZnB}$ & BS-1 & BS-3 & BS-5 & & \\
\hline \multicolumn{8}{|l|}{ (1-21 days) } \\
\hline 1 day $(\mathrm{g})$ & 47.08 & 47.32 & 47.05 & 47.49 & 47.14 & 0.172 & 0.915 \\
\hline 21 day $(g)$ & $641.6^{\mathrm{bc}}$ & $621.31^{c}$ & $646.04^{\mathrm{abc}}$ & $657.36^{a b}$ & $670.54^{a}$ & 4.164 & 0.004 \\
\hline ADFI (g/day) & 40.13 & 40.28 & 42.79 & 43.85 & 42.71 & 0.601 & 0.185 \\
\hline ADG (g/day) & $28.27^{b c}$ & $27.28^{c}$ & $28.59^{a b}$ & $29.07^{\mathrm{ab}}$ & $29.62^{a}$ & 0.22 & 0.004 \\
\hline FCR $(g / g)$ & 1.42 & 1.48 & 1.5 & 1.51 & 1.44 & 0.021 & 0.653 \\
\hline Survivability rates (\%) & 99.17 & 98.33 & 98.33 & 97.5 & 99.17 & 0.49 & 0.826 \\
\hline \multicolumn{8}{|l|}{ (21-42 days) } \\
\hline 42 day $(\mathrm{g})$ & $1600.39^{a b}$ & $1,578.24^{b}$ & $1,620.1^{\mathrm{ab}}$ & $1,652.0^{a}$ & $1,636.98^{a}$ & 8.305 & 0.041 \\
\hline ADFI (g/day) & 87.67 & 86.65 & 89.95 & 90.57 & 88.35 & 0.905 & 0.664 \\
\hline ADG (g/day) & 50.48 & 50.33 & 51.37 & 52.55 & 51.35 & 0.555 & 0.755 \\
\hline FCR $(g / g)$ & 1.74 & 1.73 & 1.75 & 1.73 & 1.72 & 0.0134 & 0.96 \\
\hline Survivability rates (\%) & 96.67 & 99.17 & 100 & 98.33 & 99.17 & 0.48 & 0.225 \\
\hline \multicolumn{8}{|l|}{$(1-42)$} \\
\hline ADFI (g/day) & 62.71 & 62.30 & 65.2 & 66.06 & 64.39 & 0.646 & 0.305 \\
\hline ADG (g/day) & 38.82 & 38.22 & 39.41 & 40.22 & 39.94 & 0.295 & 0.189 \\
\hline FCR $(g / g)$ & 1.62 & 1.63 & 1.65 & 1.64 & 1.61 & 0.01 & 0.65 \\
\hline Survivability rates (\%) & 95.83 & 97.5 & 98.33 & 95.83 & 98.33 & 0.746 & 0.710 \\
\hline
\end{tabular}

${ }^{1} A D G$, average daily body gain; $A D F l$, average daily feed intake; FCR (g/g), ADFI (g/day)/ADG (g/day).

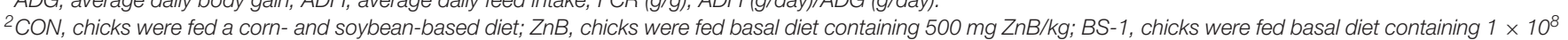

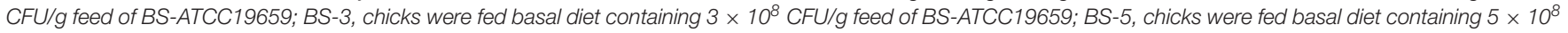
CFU/g feed of BS-ATCC19659.

$a, b, c$ Values in the same row with different letter superscripts mean significant differences $(p<0.05)$. Data are presented as the mean \pm SEM ( $n=6)$.

effect on ADG during 1-21 days $(p<0.05)$. However, there was no significant effect on ADFI, ADG, FCR, and survivability rates among the five groups (Table 2).

\section{Serum Antioxidant Assessment of Host Broiler}

The results of the serum antioxidant profile are shown in Table 3. There were significant improvements in serum activities of SOD, CAT, T-AOC, and GSH in both BS-5 and $\mathrm{ZnB}$ groups compared to the CON group $(p<0.05)$. On the other hand, the serum concentration of MDA was significantly decreased in both the BS-5 and ZnB groups ( $p<0.05$ ) (Table 3). Thus, collectively, the antioxidant defense was improved as affected by the dietary inclusion of BS-ATCC19659.

\section{Serum Immunity Assessment of Host Broiler}

The dietary administration of BS-ATCC19659 groups for 42 days on the levels of immunoglobulins, complement components, sIgA, interleukins, TNF- $\alpha$, and TGF- $\beta$ in serum of the host broiler is shown in Table 3. ZnB and BS-5 groups significantly increased the levels of immunoglobulins (IgE, IgA, and IgM) and C3, C4, sIgA, IL-10, and TGF- $\beta$ as compared with that of the CON group $(p<0.05)$; IgG was significantly higher in $\mathrm{ZnB}$ and $\mathrm{BS}-3$ groups than those in the CON group $(p<0.05)$. On the other hand, IL-4 and IL-6 were increased significantly in the serum of the
CON group compared to both BS-ATCC19659 and ZnB groups $(p<0.05)$; TNF- $\alpha$ was significantly higher in CON and BS-1 groups $(p<0.05)$ (Table 3$)$.

\section{Intestinal Histomorphometric Assessment of Host Broiler}

The effects of dietary administration of BS-ATCC19659 for 42 days on the morphology and morphometry of the ileum and jejunum in broiler are shown in Figure 1. The results showed that the heights of the villus length $(\mu \mathrm{m})$ in the sections of the ileum were significantly increased with the BS-3 and $\mathrm{ZnB}$ groups $(p<0.05)$; the villus/crypt ratio was significantly higher in the BS-5 and BS-3 groups ( $p<0.05)$ (Table 4). However, the crypt depth $(\mu \mathrm{m})$ in the sections of the jejunum was measured in both CON, BS-1, and BS-3 groups ( $p<0.05$ ); the villus/crypt ratio was significantly increased in the $\mathrm{ZnB}$ and $\mathrm{BS}-5$ groups compared to that of the CON group $(p<0.05)$. Conversely, the crypt depth and villus length in the ileum and jejunum were not affected by the treatments, respectively, as shown in Table 4.

\section{Growth, Antioxidant, and Cytokine Gene Expression Assessment of Host Broiler}

The relative mRNA expression of target genes was compared using qRT-PCR in the liver and intestinal tissues among the treatment groups following the $2^{-\Delta \Delta C T}$ method and is shown in Table 5. Regarding relative mRNA expression in liver tissue, the 
TABLE 3 | Effect of dietary supplementation of Bacillus subtilis ATCC19659 on the broiler's serum antioxidant indexes, immunoglobulin, and cytokine profile.

\begin{tabular}{|c|c|c|c|c|c|c|c|}
\hline \multirow[t]{2}{*}{ Items $^{1}$} & \multicolumn{5}{|c|}{ Treatments $^{2}$} & \multirow[t]{2}{*}{ SEM } & \multirow[t]{2}{*}{$p$-value } \\
\hline & CON & $\mathrm{ZnB}$ & BS-1 & BS-3 & BS-5 & & \\
\hline $\mathrm{SOD}(\mathrm{u} / \mathrm{mL})$ & $104.24^{c}$ & $140.34^{a}$ & $103.94^{c}$ & $120.64^{b}$ & $138.37^{a}$ & 3.440 & $<0.001$ \\
\hline CAT (U/mL) & $4.63^{b}$ & $8.47^{a}$ & $4.86^{b}$ & $7.00^{\mathrm{a}}$ & $7.45^{\mathrm{a}}$ & 0.347 & $<0.001$ \\
\hline MDA (nmol/mL) & $9.18^{a}$ & $3.10^{c}$ & $8.83^{a}$ & $7.97^{a}$ & $5.96^{\mathrm{b}}$ & 0.408 & $<0.001$ \\
\hline $\mathrm{T}-\mathrm{AOC}(\mathrm{U} / \mathrm{mL})$ & $6.56^{d}$ & $15.46^{a}$ & $5.79^{d}$ & $8.76^{c}$ & $12.45^{b}$ & 0.725 & $<0.001$ \\
\hline GSH (mg/gL) & $19.29^{d}$ & $35.86^{a}$ & $22.77^{\mathrm{cd}}$ & $28.27^{b c}$ & $32.09^{a b}$ & 1.434 & $<0.001$ \\
\hline $\lg E(\mu \mathrm{g} / \mathrm{mL})$ & $0.016^{\mathrm{C}}$ & $0.02^{a}$ & $0.017^{\mathrm{C}}$ & $0.017^{\mathrm{C}}$ & $0.02^{b}$ & 0.001 & $<0.001$ \\
\hline $\lg A(\mu \mathrm{g} / \mathrm{mL})$ & $0.51^{d}$ & $0.71^{a}$ & $0.59^{c}$ & $0.62^{b c}$ & $0.65^{b}$ & 0.013 & $<0.001$ \\
\hline $\lg G(\mu \mathrm{g} / \mathrm{mL})$ & $1.78^{\mathrm{c}}$ & $2.79^{a}$ & $1.94^{\mathrm{c}}$ & $2.35^{\mathrm{b}}$ & $2.34^{b}$ & 0.39 & $<0.001$ \\
\hline $\lg M(\mu \mathrm{g} / \mathrm{mL})$ & $0.90^{c}$ & $1.22^{\mathrm{a}}$ & $0.96^{c}$ & $1.07^{\mathrm{b}}$ & $1.09^{b}$ & 0.023 & $<0.001$ \\
\hline C3 (mg/mL) & $0.59^{b}$ & $0.77^{a}$ & $0.06^{c}$ & $0.67^{b c}$ & $0.71^{a b}$ & 0.186 & 0.002 \\
\hline C4 (mg/mL) & $0.14^{b}$ & $0.19^{a}$ & $0.16^{a b}$ & $0.17^{a b}$ & $0.19^{a}$ & 0.006 & 0.007 \\
\hline $\operatorname{slg} A(n g / m L)$ & $1.49^{c}$ & $2.17^{\mathrm{a}}$ & $1.75^{\mathrm{bc}}$ & $1.84^{b}$ & $1.9^{a b}$ & 0.056 & $<0.001$ \\
\hline IL-10 (ng/L) & $36.13^{d}$ & $63.52^{a}$ & $43.06^{\mathrm{cd}}$ & $49.72^{b c}$ & $51.89^{b}$ & 2.02 & $<0.001$ \\
\hline IL-4 (ng/L) & $159.88^{a}$ & $103.41^{d}$ & $142.87^{b}$ & $126.08^{c}$ & $115.4^{\mathrm{cd}}$ & 4.25 & $<0.001$ \\
\hline IL-6 (ng/L) & $29.34^{a}$ & $16.57^{c}$ & $23.7^{\mathrm{b}}$ & $23.11^{b}$ & $19.29^{c}$ & 0.923 & $<0.001$ \\
\hline TNF- $\alpha$ (ng/L) & $74.67^{a}$ & $50.96^{c}$ & $74.73^{a}$ & $67.06^{a b}$ & $59.03^{b c}$ & 2.104 & $<0.001$ \\
\hline TGF- $\beta$ (ng/L) & $134.32^{c}$ & $195.92^{a}$ & $144.13^{C}$ & $161.29^{b}$ & $167.16^{b}$ & 4.44 & $<0.001$ \\
\hline
\end{tabular}

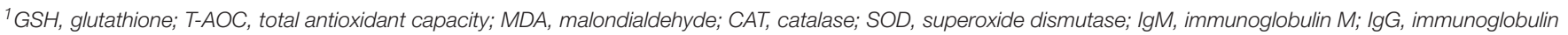

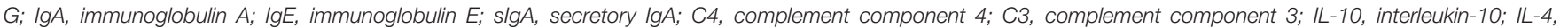

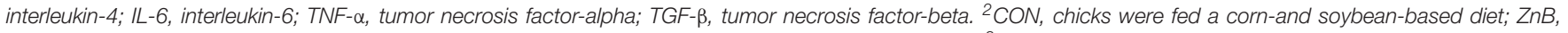

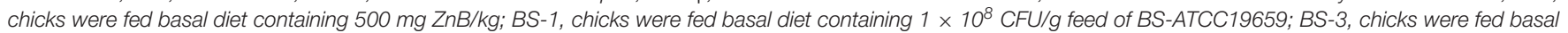
diet containing $3 \times 10^{8}$ CFU/g feed of BS-ATCC19659; BS-5, chicks were fed basal diet containing $5 \times 10^{8}$ CFU/g feed of BS-ATCC19659.

$a, b, c, d$ Values in the same row with different letter superscripts mean significant differences $(p<0.05)$. Data are presented as the mean $\pm S E M(n=6)$.

highest mRNA expressions for TGF- $\beta, I G F-1$, and $I F N-\gamma$ genes were detected in the BS-3 group compared to the CON group $(p<0.05)$; GHR and SOD genes were significantly higher in the BS-1 than CON group ( $p<0.05$ ); GPX and CAT genes were significantly increased in the BS-5 group compared to the CON group $(p<0.05)$ (Table 5$)$. In the intestinal tissue, the highest mRNA expressions for GHR, TGF- $\beta, I F N-\gamma$, and GPX genes were measured in the CON group $(p<0.05)$. Conversely, the IGF1 gene was detected in the $\mathrm{ZnB}$ and CON groups than in other groups $(p<0.05)$; CAT and SOD genes were not affected by the dietary supplementation with ZnB and BS-ATCC19659 (Table 5).

\section{Microbial Diversity Assessment of Host Broiler}

The alpha diversity indicators of observed OUT, Chao1, Shannon, Simpson, and Goods coverage indices among the treatment groups are presented in Table 6. The results of indices as affected by the dietary inclusion of $\mathrm{ZnB}$ and BS-ATCC19659 showed no significant effect $(p>0.05)$ on alpha diversity indicators (Table 6). Beta diversity indicators of PCA, PCoA, and NMDS measured the intragroup and intergroup distances and are presented in Figure 2. Statistically significant $p$-values measured the intragroup and intergroup distances and found that the differences between groups were not significant plots of PCA (Figure 2A), unweighted PCoA (Figure 2C), and weighted and unweighted NMDS (Figures 2D,E), while a significant difference was estimated in the weighted PCoA plot $(p<0.05)$ (Figure 2B).

\section{Microbial Composition Assessment of Host Broiler}

The effect of the different levels of BS-ATCC19659 groups as a dietary supplement on different phylum and genus taxa levels in the cecal microbial composition is shown in Figure 3. The analysis of the phylum level revealed that Firmicutes and Bacteroidetes were the predominant phyla of the cecal community, accounting for approximately 75.5 and $19.7 \%$, respectively (Figure $3 \mathbf{A}$ ). The relative abundance of Cyanobacteria phyla was significantly decreased in the $\mathrm{ZnB}$ and BS-1 groups compared to the CON group $(p<0.05)$, as shown in Table 7.

Regarding genus taxa level, Faecalibacterium, Alistipes, and Ruminococcaceae_UCG-014 were the dominant genera of the cecal community, accounting for approximately 12.46, 7.54, and $6.59 \%$, respectively (Figure 3B). The relative abundance of the CHKCI001 genus was significantly increased in the CON group as compared to the $\mathrm{ZnB}$ and BS-ATCC19659 groups $(p<0.05)$. The relative abundance of GCA-900066575 (Lachnospiraceae) was significantly increased in the BS-5 and BS-3 groups compared to the CON group $(p<0.05)$; the relative abundance of Papillibacter was significantly higher in the BS-3 and $\mathrm{ZnB}$ groups than in the CON group $(p<0.05)$. However, the relative abundance of Anaerofustis was significantly lower in the BS-1 and $\mathrm{ZnB}$ groups than in other groups $(p<0.05)$. The relative abundance of Escherichia-Shigella was significantly reduced in the BS-3 group as compared to other treatments $(p<0.05)$; 


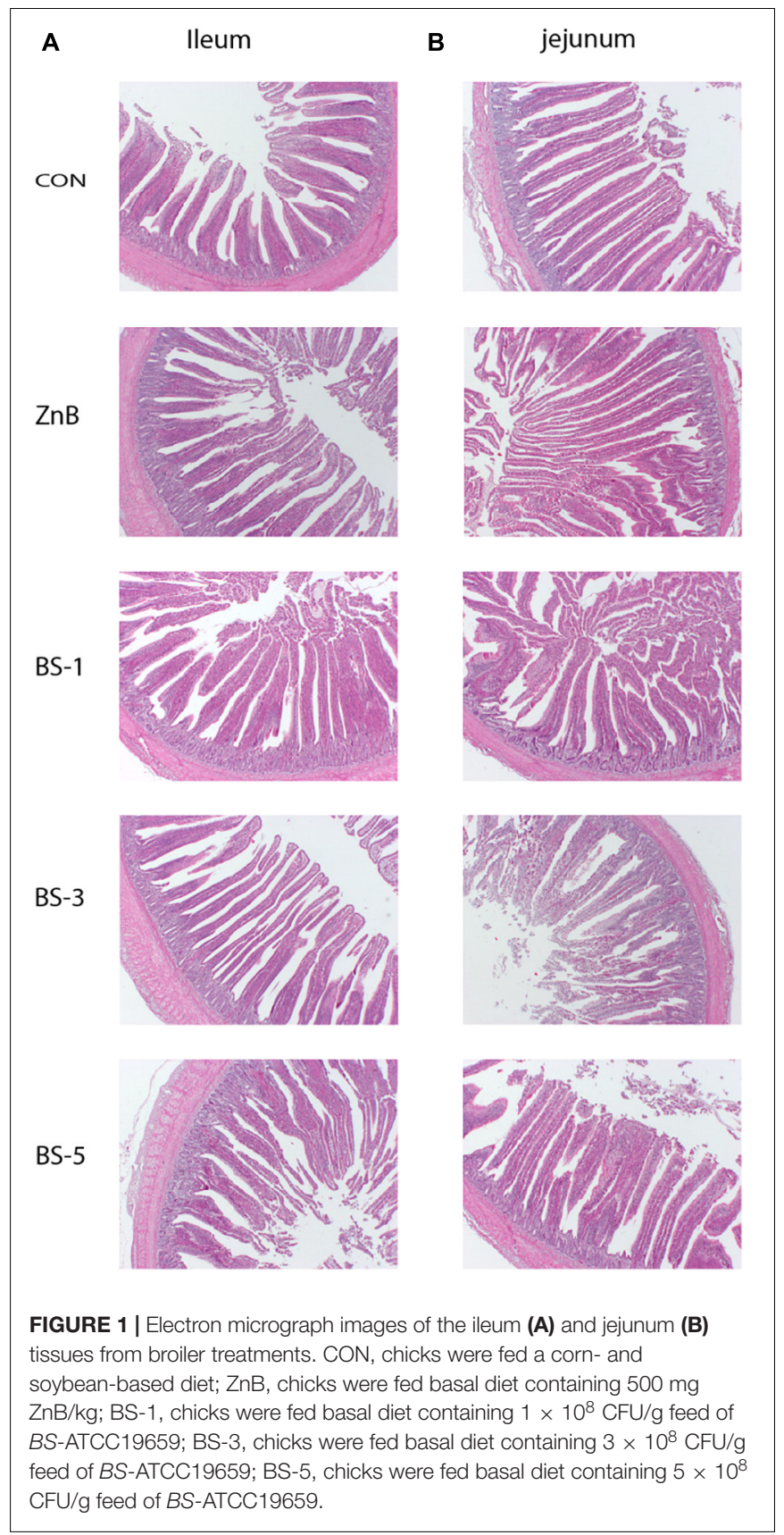

the relative abundance of Clostridia_unclassified was significantly higher in CON $(p<0.05)$ than in other treatments. The relative abundance of Lactobacillus in the BS-1 group significantly decreased compared with the other treatments $(p<0.05)$. However, the $\mathrm{ZnB}$ group tended to increase in the relative abundance of Lactobacillus, as shown in Table 7.

\section{DISCUSSION}

In the present study, it was found that adding 3 and $5 \times 10^{8}$ CFU of BS-ATCC19659/g feed for 42 days helped improve broiler performance (growth performance, antioxidant status, immunity, and gastrointestinal responses) by colonizing a distinct GM community in the gastrointestinal tract, resulting in improved gut health for broiler productivity. These findings could be a suitable alternative to the use of $\mathrm{ZnB}$ antibiotics in organic poultry production. Precisely, probiotic products are widely used in several commercial applications, especially Bacillus subtilis strains that support digestion, healthy GM, and immune response, as an alternative to an antibiotic in poultry diets (Gao et al., 2017; Qin et al., 2018).

In the present study, BS-5 and BS-3 groups improved body weight at the $21^{\text {st }}$ and $42^{\text {nd }}$ days and ADG during 1-21 days. They showed consistency with the findings of Bai et al. (2017), who tested the inclusion of Bacillus subtilis and found an increase in broiler growth performance, providing beneficial effects on broiler growth and potentially replacing antibiotic growth promoters (Park et al., 2020). In broiler chickens, some certain strains of Bacillus subtilis such as Bacillus subtilis B10 (Qin et al., 2018), Bacillus subtilis 29784 (Jacquier et al., 2019), Bacillus subtilis DSM 32315 (Bortoluzzi et al., 2018), Bacillus subtilis BYS2 (Dong et al., 2020), Bacillus subtilis GM5 (Hadieva et al., 2021), and Bacillus subtilis PB6 (Abramowicz et al., 2019) have been suggested to promote growth activities and healthy GM. The expression of mRNA GHR and mRNA IGF-1 were increased in the BS-ATCC19659 (BS-1 and BS-3) groups in the liver. IGF-1 and GHR genes play a critical role in the growth rate of chicken. Thus, the improved effect of the BS-3 group may be linked to the alteration in genes in broiler-related growth (Kareem et al., 2016). However, other researchers illustrated that dietary supplementation of probiotics sometimes had minimal or no effect on growth performance. This may be due to strains of probiotic bacteria, preparation methods, administration, dosage, composition, hygiene status, and bird age (Lee et al., 2010; Zhang et al., 2012).

The shift in balance between oxidants [such as reactive oxygen species (ROS) and free radicals] and antioxidants in favor of oxidants is termed "oxidative stress." Oxidative stress severely affects Broiler chickens, damaging nucleic acids, cell molecules, and mitochondrial membranes. Several studies reported that additional probiotics containing Bacillus spp. in diet enhanced oxidative resistance and increased exogenous antioxidant capacity in broiler chickens (Bai et al., 2017; Abramowicz et al., 2019; Dong et al., 2020). Both BS-5 and $\mathrm{ZnB}$ groups had a significant increase in GSH, T-AOC, CAT, and SOD activities from the results obtained. They decreased the MDA concentration in the entire period in serum at 42 days. The relative mRNA expressions of antioxidant genes including SOD, CAT, and GPX were significantly increased in BS-ATCC19659 groups (BS-1 and BS-5) in liver tissue. The results of Zhang et al. (2017) were in agreement that the dietary Bacillus species in broilers improved significantly in both GSH, SOD, and CAT activities in serum. Likewise, dietary Bacillus subtilis improved the antioxidant capacity in broiler chickens (Dong et al., 2020). He et al. (2019) demonstrated that probiotics had a positive role in oxidation resistance, scavenging ROS and stimulating antioxidant capability. The animal defense system can depend on 
TABLE 4 | Effect of dietary supplementation of Bacillus subtilis ATCC19659 on intestinal morphology of the ileum and jejunum in broilers.

\begin{tabular}{|c|c|c|c|c|c|c|c|}
\hline \multirow[t]{2}{*}{ Items } & \multicolumn{5}{|c|}{ Treatments $^{1}$} & \multirow[t]{2}{*}{ SEM } & \multirow[t]{2}{*}{$P$-value } \\
\hline & CON & $\mathrm{ZnB}$ & BS-1 & BS-3 & BS-5 & & \\
\hline \multicolumn{8}{|l|}{ Ileum } \\
\hline Villus length ( $\mu \mathrm{m})$ & $598.33^{C}$ & $759.20^{a}$ & $679.95^{\mathrm{bc}}$ & $839.46^{a}$ & $693.28^{\mathrm{abc}}$ & 23.32 & 0.015 \\
\hline Crypt depth ( $\mu \mathrm{m})$ & 116.7 & 124.6 & 131.45 & 133.31 & 118.59 & 3.767 & 0.544 \\
\hline Villus height/crypt dept & $5.21^{b}$ & $6.12^{\mathrm{ab}}$ & $5.26^{b}$ & $7.47^{\mathrm{a}}$ & $6.78^{a b}$ & 0.241 & 0.009 \\
\hline \multicolumn{8}{|l|}{ Jejunum } \\
\hline Villi length ( $\mu \mathrm{m})$ & 934.25 & 1033.17 & 830.64 & 843.71 & 874.92 & 24.10 & 0.483 \\
\hline Crypt depth ( $\mu \mathrm{m})$ & $152.83^{a}$ & $117.06^{b}$ & $161.9^{a}$ & $152.71^{\mathrm{a}}$ & $140.27^{a b}$ & 4.01 & 0.004 \\
\hline Villus height/crypt dept & $6.53^{\mathrm{bc}}$ & $9.44^{a}$ & $5.21^{\mathrm{c}}$ & $5.98^{\mathrm{bc}}$ & $6.89^{b}$ & 0.256 & $<0.001$ \\
\hline
\end{tabular}

${ }^{1}$ CON, chicks were fed a corn-and soybean-based diet; ZnB, chicks were fed basal diet containing $500 \mathrm{mg} Z \mathrm{ZnB} / \mathrm{kg}$; BS-1, chicks were fed basal diet containing $1 \times 10^{8}$ CFU/g feed of BS-ATCC19659; BS-3, chicks were fed basal diet containing $3 \times 10^{8}$ CFU/g feed of BS-ATCC19659; BS-5, chicks were fed basal diet containing $5 \times 10^{8}$ CFU/g feed of BS-ATCC19659.

$a, b, c$ Values in the same row with different letter superscripts mean significant differences $(p<0.05)$. Data are presented as the mean \pm SEM $(n=6)$.

TABLE 5 | Effects of dietary supplementation of Bacillus subtilis ATCC19659 on the mRNA abundance of GHR, TGF- $\beta$, IGF-1, IFN- $\gamma$, SOD, CAT, and GPX in liver and intestine tissue of broiler.

\begin{tabular}{|c|c|c|c|c|c|c|c|}
\hline \multirow[t]{2}{*}{ Treatments $^{2}$} & \multicolumn{6}{|c|}{ Items $^{1}$} & \multirow[b]{2}{*}{$G P X$} \\
\hline & GHR & TGF- $\beta$ & IGF-1 & $I F N-\gamma$ & $S O D$ & $C A T$ & \\
\hline \multicolumn{8}{|l|}{ Liver } \\
\hline CON & $1.12^{b}$ & $1.03^{b}$ & $1.04^{b}$ & $1.13^{b}$ & $1.13^{b}$ & $1.09^{b}$ & $1.02^{b}$ \\
\hline $\mathrm{ZnB}$ & $0.5^{\mathrm{c}}$ & $1.02^{b}$ & $0.99^{b}$ & $1.49^{b}$ & $1.14^{b}$ & $1.65^{b}$ & $1.02^{b}$ \\
\hline$B S-1$ & $2.35^{a}$ & $0.58^{\mathrm{c}}$ & $1.34^{b}$ & $1.05^{b}$ & $8.23^{a}$ & $5.01^{a}$ & $1.32^{a}$ \\
\hline BS-3 & $2.22^{a}$ & $1.46^{\mathrm{a}}$ & $2.12^{\mathrm{a}}$ & $2.4^{\mathrm{a}}$ & $7.47^{\mathrm{a}}$ & $4.81^{a}$ & $1.07^{b}$ \\
\hline$B S-5$ & $1.3^{\mathrm{b}}$ & $1.44^{\mathrm{a}}$ & $1.88^{a}$ & $1.61^{b}$ & $1.51^{b}$ & $5.45^{a}$ & $1.42^{a}$ \\
\hline SEM & 0.16 & 0.08 & 0.116 & 0.123 & 0.672 & 0.393 & 0.047 \\
\hline$p$-value & $<0.001$ & 0.001 & $<0.001$ & $<0.001$ & $<0.001$ & $<0.001$ & 0.002 \\
\hline \multicolumn{8}{|l|}{ Intestinal } \\
\hline $\mathrm{CON}$ & $1.09^{a}$ & $1.22^{\mathrm{a}}$ & $1.1^{\mathrm{a}}$ & $1.17^{\mathrm{a}}$ & 1.09 & 1.09 & $1.16^{\mathrm{a}}$ \\
\hline $\mathrm{ZnB}$ & $0.63^{b}$ & $0.76^{b}$ & $1.17^{\mathrm{a}}$ & $0.92^{b}$ & 0.9 & 0.84 & $0.64^{b}$ \\
\hline$B S-1$ & $0.54^{b}$ & $0.80^{b}$ & $0.45^{b}$ & $0.78^{b}$ & 1.13 & 1.2 & $0.61^{b}$ \\
\hline BS-3 & $0.57^{b}$ & $0.72^{b}$ & $0.59^{b}$ & $0.85^{b}$ & 0.96 & 0.77 & $0.62^{b}$ \\
\hline BS-5 & $0.49^{b}$ & $0.38^{\mathrm{c}}$ & $0.44^{b}$ & $0.68^{b}$ & 1.23 & 0.8 & $0.57^{b}$ \\
\hline SEM & 0.049 & 0.071 & 0.073 & 0.05 & 0.088 & 0.063 & 0.07 \\
\hline$p$-value & $<0.001$ & 0.001 & $<0.001$ & 0.006 & 0.717 & 0.09 & 0.02 \\
\hline
\end{tabular}

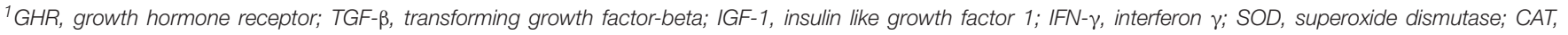
catalase; GPX, glutathione peroxidase.

${ }^{2} \mathrm{CON}$, Chicks were fed a corn-and soybean-based diet; ZnB, chicks were fed basal diet containing $500 \mathrm{mg} Z \mathrm{ZnB} / \mathrm{kg}$; BS-1, chicks were fed basal diet containing $1 \times 10^{8}$ CFU/g feed of BS-ATCC19659; BS-3, chicks were fed basal diet containing $3 \times 10^{8}$ CFU/g feed of BS-ATCC19659; BS-5, chicks were fed basal diet containing $5 \times 10^{8}$ feed CFU/g of BS-ATCC19659.

$a, b, c$ Values in the same row with different letter superscripts mean significant differences $(p<0.05)$. Data are presented as the mean \pm SEM ( $n=5)$.

probiotics as a natural source for protection of the oxidative stress result from ROS.

Immunoglobulins and complement components are parameters that reflect the immune status of animals because it plays crucial roles in the immune system (Rajput and $\mathrm{Li}$, 2012). The application of probiotics stimulates immune cells and cytokine production. Our findings revealed that BS-5 and $\mathrm{ZnB}$ groups improved serum immunoglobulins (IgM, IgA, and IgE), secretory $\operatorname{IgA}(\operatorname{IgA})$, and complement components (C3 and C4). The results agree with Bai et al. (2017), who reported that Bacillus subtilis fmbJ increased the serum IgA and IgG concentrations of broiler chicken at 42 days. Also, Sun et al. (2011) reported that serum C4 and C3 were increased with the addition of Psychrobacter spp. in the broiler diet. Likewise, the Bacillus subtilis probiotic increased the serum sIgA and $\lg \mathrm{A}$ of broiler chickens (Rajput et al., 2017). sIgA works on the sustenance of mucosal homeostasis that influences the intestinal microbiota content and improves the immune system (Lammers et al., 2010; Wang et al., 2017; Liu et al., 2019). In the present study, serum interleukin (IL-10) and tumor 
TABLE 6 | Effect of dietary supplementation of Bacillus subtilis ATCC19659 on cecal microbiota communities of broilers by alpha diversity measures.

\begin{tabular}{|c|c|c|c|c|c|c|c|}
\hline \multirow[t]{2}{*}{ Items } & \multicolumn{5}{|c|}{ Treatments $^{a}$} & \multirow[t]{2}{*}{ SEM } & \multirow[t]{2}{*}{$p$-value } \\
\hline & CON & $\mathrm{ZnB}$ & BS-1 & BS-3 & BS-5 & & \\
\hline Chao1 & 525.62 & 558.98 & 555.19 & 557.43 & 506.12 & 18.687 & 0.881 \\
\hline Shannon & 6.62 & 6.98 & 6.75 & 6.97 & 6.83 & 0.085 & 0.661 \\
\hline Simpson & 0.97 & 0.98 & 0.97 & 0.98 & 0.98 & 0.003 & 0.618 \\
\hline
\end{tabular}

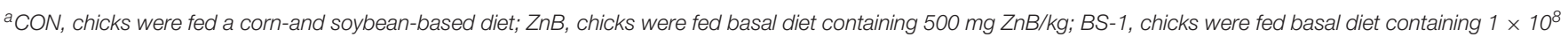

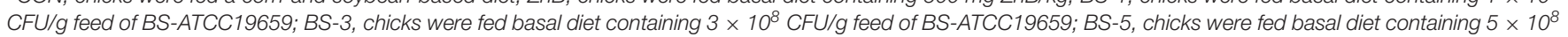
CFU/g feed of BS-ATCC19659.

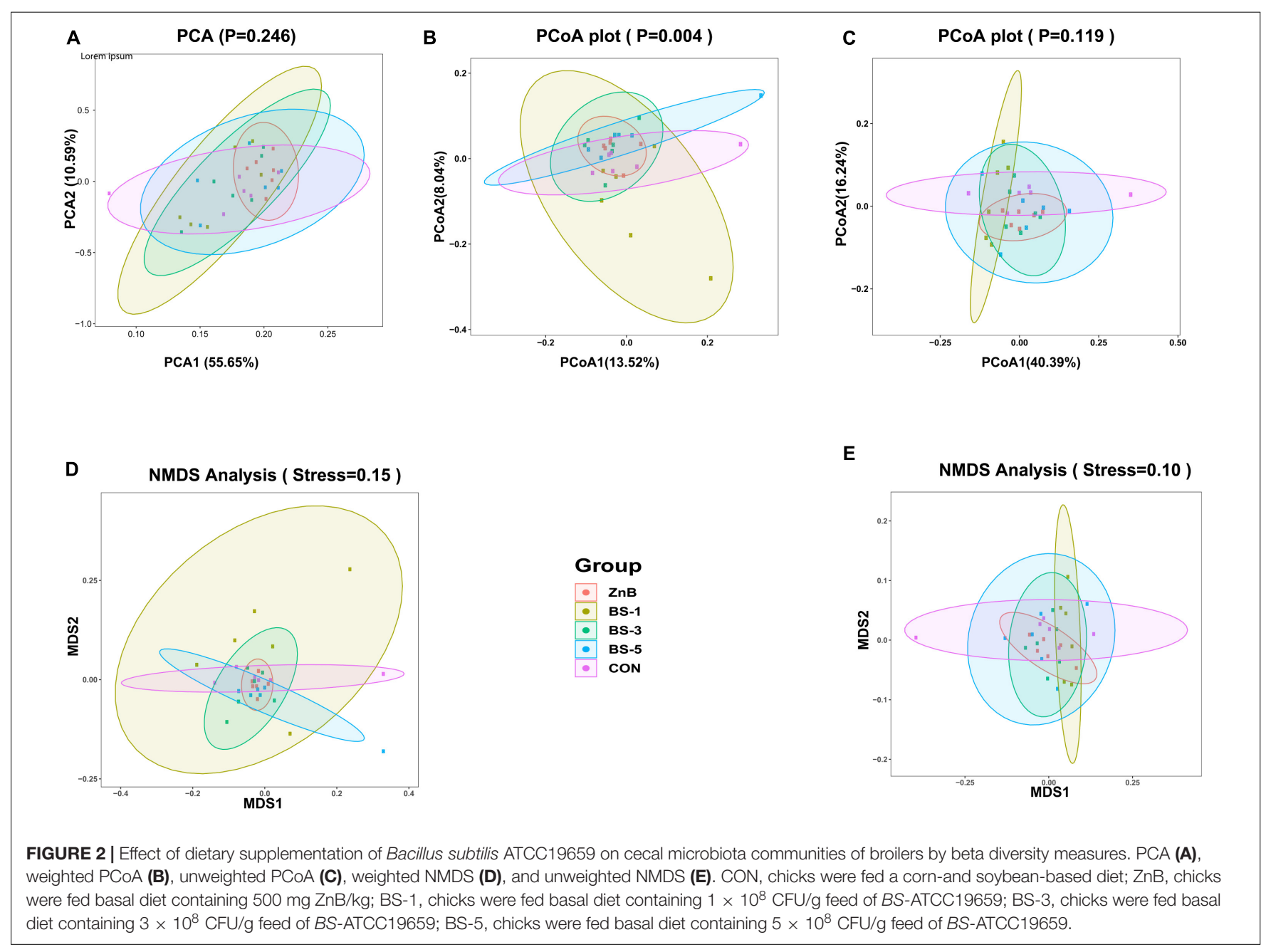

necrosis factor-beta (TGF- $\beta$ ) were enhanced in BS-5 and $\mathrm{ZnB}$ groups, but serum IL-6, IL-4, and TNF- $\alpha$ were increased in the CON group and BS-1 groups. The mRNA expressions of TGF- $\beta$ and IFN- $\gamma$ were significantly increased with the BS-3 group in liver tissue. IFN- $\gamma$ is a standard indicator of cellular immunity through the higher levels and has been linked with enhanced protective immune (Lee et al., 2014). TGF- $\alpha$ and IL-10 stimulate class switching of B cells to create IgA that proliferates and activates by interactions with $\mathrm{T}$ cells (Bos et al., 2001;
Azad et al., 2018). This result agrees with Selvam et al. (2009) who observed that the inclusion of Bacillus subtilis PB6 had significantly higher levels of anti-inflammatory cytokines (TGF- $\beta$ and IL-10) in plasma and significantly decreased the levels of pro-inflammatory cytokines (IL-6 and TGF- $\alpha$ ) because it is found to secrete surfactins of cyclic lipopeptides (antibacterial). These surfactins prevent phospholipase A2. The phospholipase $\mathrm{A} 2$ is a rate-limiting enzyme that takes part in the arachidonic acid linked with the inflammatory pathway. Conversely, 
A

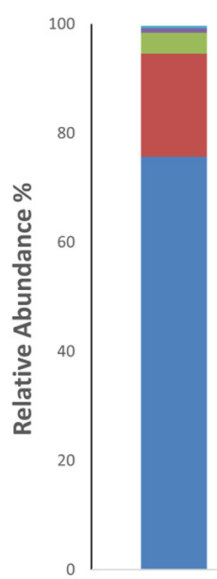

CON
Phylum Taxa

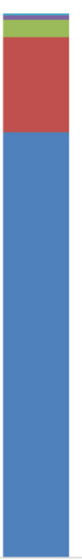

$\mathrm{ZnB}$

BS-1

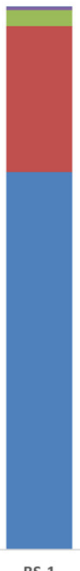

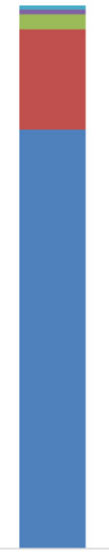

BS-3

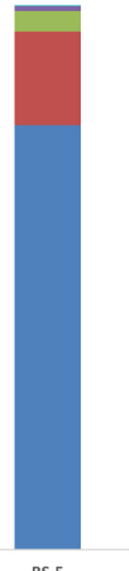

- Firmicutes

- Bacteroidetes

- Proteobacteria

- Tenericutes

Verrucomicrobia
B

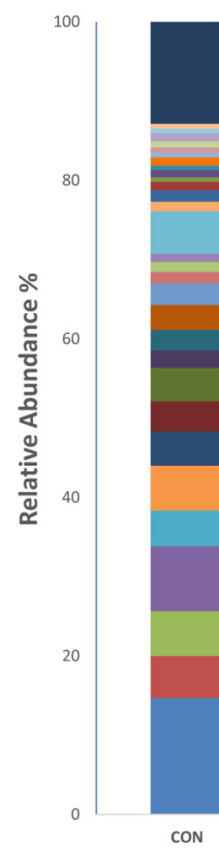

Genus Taxa

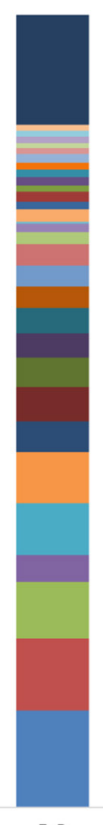

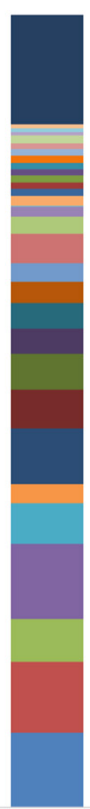

BS-1

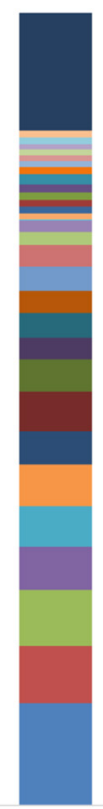

BS-3

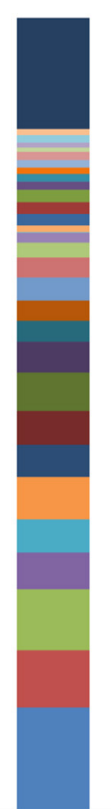

- Faecalibacte
- Alistipes

Ruminococcaceae_UCG-014

- Parabacteroides

- Ruminococcaceae_UCG-005

n Lactobacillus

- Bacteroides

- Lachnospiraceae_unclassified

- Intestinimonas

- Ruminococcaceae unclassified

- Clostridiales_vadinBB60_group_unclassifiec

- Firmicutes_unclassified

- Ruminiclostridium_9

- Ruminococcus]_torques_group

= Clostridiales_unclassified

- Butyricimonas

- Romboutsia

= Phascolarctobacterium

- Parasutterella

- Erysipelatoclostridium

- Eubacterium]_coprostanoligenes_group

- Oscillibacter

- Ruminococcaceae_NK4A214_group

- Christensenellaceae_R-7_group

- Clostridium

a Bilophila

in Butyricicoccus

- Thalassospira

- GCA-900066575

= Flavonifractor

- Others

FIGURE 3 | Effect of dietary supplementation of Bacillus subtilis ATCC19659 on the relative abundance of cecal microbiota communities at phylum (A) and genus (B). CON, chicks were fed a corn- and soybean-based diet; ZnB, chicks were fed basal diet containing 500 mg ZnB/kg; BS-1, chicks were fed basal diet containing $1 \times 10^{8} \mathrm{CFU} / \mathrm{g}$ feed of BS-ATCC19659; BS-3, chicks were fed basal diet containing $3 \times 10^{8} \mathrm{CFU} / \mathrm{g}$ feed of BS-ATCC19659; BS-5, chicks were fed basal diet containing $5 \times 10^{8} \mathrm{CFU} / \mathrm{g}$ feed of BS-ATCC19659.

Zhang et al. (2016) discovered that supplementation of Clostridium butyricum changed the sensitization of the host by increasing the concentrations of IL-6, IL-8, and TNF- $\alpha$. Also, Rajput et al. (2013) reported that dietary supplementation with Bacillus subtilis increased IL- 6 and TFN- $\alpha$ compared to a control group in the ileum and jejunum. Huang et al. (2012) reported an increase in the concentration of inflammatory cytokines. These might be due to different probiotics species.

The dietary Bacillus subtilis supplementation enhances the absorption surface because it improves the crypt cell proliferation in the small intestinal and Bacillus spp. can also colonize and form niches in the intestine protected from pathogens and increase 
TABLE 7 | Effect of dietary supplementation of Bacillus subtilis ATCC19659 on bacterial taxonomy within the cecal contents of broilers.

\begin{tabular}{|c|c|c|c|c|c|c|c|}
\hline \multirow[t]{2}{*}{ Items } & \multicolumn{5}{|c|}{ Relative abundance (\%) } & \multirow[t]{2}{*}{ SEM } & \multirow[t]{2}{*}{$p$-value } \\
\hline & ${ }^{1} \mathrm{CON}$ & $\mathrm{ZnB}$ & BS-1 & BS-3 & BS-5 & & \\
\hline \multicolumn{8}{|l|}{ Phylum } \\
\hline Cyanobacteria & $0.1^{a}$ & $0.02^{b}$ & $0.02^{b}$ & $0.08^{a b}$ & $0.03^{b}$ & 0.01 & 0.047 \\
\hline \multicolumn{8}{|l|}{ Genus } \\
\hline CHKCI001 & $0.51^{a}$ & $0.19^{b}$ & $0.13^{b}$ & $0.29^{a b}$ & $0.18^{b}$ & 0.038 & 0.02 \\
\hline GCA-900066575 & $0.54^{b}$ & $0.75^{\mathrm{ab}}$ & $0.48^{b}$ & $0.87^{\mathrm{a}}$ & $0.93^{a}$ & 0.05 & 0.01 \\
\hline Anaerofustis & $0.005^{a}$ & $0.004^{a b}$ & $0^{\mathrm{b}}$ & $0.006^{a}$ & $0.009^{a}$ & 0.001 & 0.03 \\
\hline Papillibacter & $0.019^{a b}$ & $0.032^{a}$ & $0.007^{b}$ & $0.039^{a}$ & $0.031^{a}$ & 0.004 & 0.03 \\
\hline Escherichia-Shigella & $0.64^{a}$ & $0.36^{a b}$ & $0.82^{\mathrm{a}}$ & $0.09^{b}$ & $0.23^{a b}$ & 0.11 & 0.04 \\
\hline Clostridia_unclassified & $0.01^{\mathrm{a}}$ & $0^{b}$ & $0.006^{a b}$ & $0.002^{b}$ & $0^{b}$ & 0.002 & 0.04 \\
\hline Lactobacillus & $5.66^{a}$ & $6.45^{a}$ & $2.40^{\mathrm{b}}$ & $5.25^{a}$ & $5.37^{a}$ & 0.71 & 0.04 \\
\hline
\end{tabular}

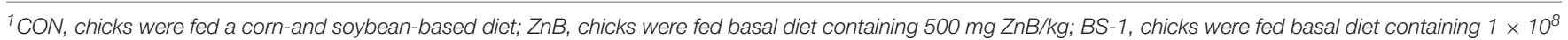

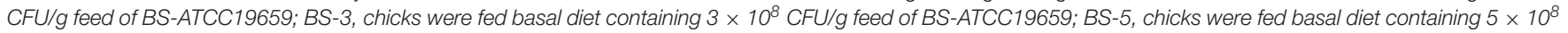
CFU/g feed of BS-ATCC19659.

$a, b, c$ Values in the same row with different letter superscripts mean significant differences $(p<0.05)$. Data are presented as the mean \pm SEM ( $n=6)$.

villus growth (Li et al., 2019). This result agrees with Deng et al. (2012), who reported that Bacillus licheniformis of dietary supplementation significantly increased the villus length/crypt depth ratio of the ileum at 6 days. In addition, Bacillus coagulans TBC169 significantly increased the villus length/crypt depth ratio of the jejunum (21 $1^{\text {st }}$ and $42^{\text {nd }}$ days) and ileum (42 days) (Li et al., 2019). Thus, Bacillus spp. might improve the absorptive surface of the jejunum and ileum, which is attributed to the developing proliferation of crypt cells in the small intestine and improves the growth performance in the broiler (He et al., 2019).

The indexes of Simpson and Shannon express a diversity of the microbiome community, while the chaol index and observed species can reflect the richness of microbial diversity (Hagerty et al., 2020). PCA, PCoA, and NMDS analysis expressed that the antibiotic (ZnB) and BS-ATCC19659 groups were similar in the current experiment, and this result is in agreement with Hong et al. (2019) who reported that the NMDS was similar between the antibiotic group (chlortetracycline) and Bacillus amyloliquefaciens TL group. However, Ma et al. (2018) found that PCoA analysis separated community microbes between groups (control and Bacillus subtilis DSM 32315), and PCA analysis explained $33 \%$ of microbial diversity.

The intestinal microbiota plays several roles in intestinal morphology, immunity, nutrient absorption and metabolism, and host health (Hong et al., 2019). In this study, Firmicutes and Bacteroidetes were the predominant phylum, accounting for more than $90 \%$ of chickens. It is worth stating that some studies indicated that the dominant phylum of the cecal community is Firmicutes and Bacteroidetes in chickens (Danzeisen et al., 2011; Wei et al., 2013; Shaufi et al., 2015). Nevertheless, the dominant bacteria may change at the phylum level because of breed, age, and regional variances of selected chickens. The relative abundance of Cyanobacteria phyla decreased in $\mathrm{ZnB}$ and BS-1 groups compared to the CON group. The result of Trela et al. (2020) agrees that the inclusion of Bacillus licheniformis decreased Cyanobacteria in cecal content microbiota. Cyanobacteria phyla had some species that can produce some neurotoxins that cause diseases (Codd et al., 2005). Bacillus spp. enhanced the immunological intestinal mucosa function and regulated the bacterial flora in the intestine (Li et al., 2019).

According to our result, Faecalibacterium, Alistipes, and Ruminococcaceae_UCG-014 were the major bacterial genera in the cecum community. This result agrees with Xiao et al. (2017) and Zhu et al. (2020), who reported that Alistipes, Ruminococcin, and Faecalibacterium were the predominant genus of bacteria in the cecal community. The relative abundance of genera GCA-900066575 (Lachnospiraceae), Anaerofustis, and Papillibacter tended to increase in BS-ATCC19659 groups. These genera belong to Firmicutes, which is associated with increased weight gain in chickens by producing molecules that absorb them from the host gut wall directly as a source of energy (Chen and $\mathrm{Yu}, 2020$; Adewole and Akinyemi, 2021; Dauksiene et al., 2021). Similarly, Jumpertz et al. (2011) stated that increasing the rate of nutritional absorption in feces was associated with increased Firmicutes. In this study, the proliferation of potentially pathogenic bacteria and the relative abundance of the genera Escherichia_Shigella and Clostridia_unclassified were inhibited by $\mathrm{ZnB}$ and BSATCC19659 (BS-3, and BS-5) groups in the cecal community. Consistent with our results, $\mathrm{Li}$ et al. (2020) reported that fermented soybean meal used in broiler diet significantly reduced the relative abundance of genera Escherichia-Shigella and Clostridiales. Escherichia-Shigella as opportunistic pathogenic bacteria were described to destroy the intestinal structure and estimate pro-inflammatory activities by variable ways such as propagating the virulence factors, leading to an increase in the risk of infection and diarrhea of host ( $\mathrm{Ma}$ et al., 2018). Clostridia_unclassified can compete and interact with other microbiota for proliferation in the intestine. Clostridia_unclassified produces some toxins that cause serious poultry diseases (Bortoluzzi et al., 2019). Bacillus spp. create 
bacteriocins, which have been stated to display antibacterial function in various models (Riazi et al., 2009; Nobutani et al., 2017). Meanwhile, Bacillus spp. produce is related to lactic acid as well as other organic acid production. Lactic acid can suppress gut colonization of harmful bacteria (Cui et al., 2005). Recently, Bacillus spp. produced dysprosium, which has been identified to display a broad antibacterial spectrum (Li et al., 2019). Also, Bacillus spp. can maintain the balance of GM via converting polysaccharides to oligosaccharides (Zheng et al., 2011). The relative abundance of genus lactobacillus improved among treatments when compared to the BS-1 group. Lactobacillus is one of the essential probiotic bacteria because it improves growth performance and helps maintain the microbial balance in the intestine, suppresses harmful pathogens, and stimulates the growth of beneficial bacteria (Shokryazdan et al., 2017).

\section{CONCLUSION}

In conclusion, dietary supplementation with the BS-5 group (Bacillus subtilis ATCC19659 $5 \times 10^{8} \mathrm{CFU} / \mathrm{g}$ feed) significantly increased bodyweight, improved antioxidant capacity, enhanced immune response, and absorption of nutrition in the present experiment. Furthermore, Bacillus subtilis ATCC19659 affected cecal microbial composition. Therefore, we conclude that feeding a probiotic additive Bacillus subtilis ATCC19659 in the poultry industry might be an encouraging alternative to antibiotic growth promoters.

\section{DATA AVAILABILITY STATEMENT}

The 16sRNA sequencing raw data is available at NCBI by accession number PRJNA807482 https://www.ncbi.nlm.nih.gov/ bioproject/?term=PRJNA807482. The rest of the raw data supporting the conclusions of this article will be made available by the authors, without undue reservation.

\section{REFERENCES}

Abramowicz, K., Krauze, M., and Ognik, K. (2019). The effect of a probiotic preparation containing Bacillus subtilis PB6 in the diet of chickens on redox and biochemical parameters in their blood. Ann. Anim. Sci. 19, 433-451. doi: 10.2478/aoas-2018-0059

Adewole, D., and Akinyemi, F. (2021). Gut microbiota dynamics, growth Performance, and gut morphology in broiler chickens fed diets varying in energy density with or without bacitracin methylene disalicylate (BMD). Microorganisms 9:787. doi: 10.3390/microorganisms 9040787

Amerah, A. M., Quiles, A., Medel, P., Sánchez, J., Lehtinen, M. J., and Gracia, M. I. (2013). Effect of pelleting temperature and probiotic supplementation on growth performance and immune function of broilers fed maize/soybased diets. Anim. Feed Sci. Technol. 180, 55-63. doi: 10.1016/j.anifeedsci.2013. 01.002

Azad, M., Kalam, A., Sarker, M., and Wan, D. (2018). Immunomodulatory effects of probiotics on cytokine profiles. Biomed. Res. Int. 2018:8063647. doi: 10.1155/ 2018/8063647

Bai, K., Huang, Q., Zhang, J., He, J., Zhang, L., and Wang, T. (2017). Supplemental effects of probiotic Bacillus subtilis $\mathrm{fmbJ}$ on growth performance, antioxidant capacity, and meat quality of broiler chickens. Poult. Sci. 96, 74-82. doi: 10. 3382/ps/pew246

\section{ETHICS STATEMENT}

The License of Experimental Animals (SYXK 2014-0002) of the Animal Experimentation Ethics Committee of Southwest University, Chongqing, China, and birds were raised following the guidelines described by the Animal Care Committee of Chongqing, China. In addition, efforts were made to reduce animal suffering and were carried out in compliance with the "ARRIVE" guidelines for reporting in vivo experiments in animal research.

\section{AUTHOR CONTRIBUTIONS}

TM: investigation, data curation, methodology, formal analysis, and writing-original draft. WS and GB: investigation and methodology. AE, KM, and RZ: writing-review and editing. $\mathrm{PH}$ and LW: conceptualization and supervision. ZT: investigation, project administration, funding acquisition, and writingreviewing and editing. All authors contributed to the article and approved the submitted version.

\section{FUNDING}

This study was funded by the Chongqing Key Innovation Project for Overseas Students (cx2017024), the National Natural Science Foundation of China (31772610), the Chongqing Natural Science Foundation (cstc2021jcyj-msxmX0966), and the Hunan Institute of Microbiology (FJ2021249, F20202048).

\section{SUPPLEMENTARY MATERIAL}

The Supplementary Material for this article can be found online at: https://www.frontiersin.org/articles/10.3389/fmicb. 2021.798350/full\#supplementary-material

Bortoluzzi, C., Rothrock, M. J., Vieira, B. S., Mallo, J. J., Puyalto, M., Hofacre, C., et al. (2018). Supplementation of protected sodium butyrate alone or in combination with essential oils modulated the cecal microbiota of broiler chickens challenged with coccidia and Clostridium perfringens. Front. Sustain. Food Syst. 2:72. doi: 10.3389/fsufs.2018.00072

Bortoluzzi, C., Serpa Vieira, B., de Paula Dorigam, J. C., Menconi, A., Sokale, A., Doranalli, K., et al. (2019). Bacillus subtilis DSM 32315 supplementation attenuates the effects of Clostridium perfringens challenge on the growth performance and intestinal microbiota of broiler chickens. Microorganisms 7:71. doi: 10.3390/microorganisms7030071

Bos, N. A., Jiang, H. Q., and Cebra, J. J. (2001). T cell control of the gut IgA responseagainst commensal bacteria. Gut 48, 762-764. doi: 10.1136/gut.48.6. 762

Cammarota, G., Ianiro, G., Bibbo, S., and Gasbarrini, A. (2014). Gut microbiota modulation: probiotics, antibiotics or fecal microbiota transplantation? Intern. Emerg. Med. 9, 365-373. doi: 10.1007/s11739-014-1069-4

Chen, Y. C., and Yu, Y. H. (2020). Bacillus licheniformis-fermented products improve growth performance and the fecal microbiota community in broilers. Poult. Sci. 99, 1432-1443. doi: 10.1016/j.psj.2019.10.061

Codd, G. A., Morrison, L. F., and Metcalf, J. S. (2005). Cyanobacterial toxins: risk management for health protection. Toxicol. Appl. Pharmacol. 203, 264-272. doi: 10.1016/j.taap.2004.02.016 
Crisol-Martínez, E., Stanley, D., Geier, M. S., Hughes, R. J., and Moore, R. J. (2017). Understanding the mechanisms of zinc bacitracin and avilamycin on animal production: linking gut microbiota and growth performance in chickens. Appl. Microbiol. Biotechnol. 101, 4547-4559. doi: 10.1007/s00253-017-8193-9

Cui, Y. L., Run, S. C., and Wan, F. C. (2005). Bacteriostasis of Bacillus coagulans TBC 169 to enteropathogenic bacteria. Chin. J. Microecol. 17, 333-338.

Danzeisen, J. L., Kim, H. B., Isaacson, R. E., Tu, Z. J., and Johnson, T. J. (2011). Modulations of the chicken cecal microbiome and metagenome in response to anticoccidial and growth promoter treatment. PLoS One 6:e27949. doi: 10.1371/ journal.pone.0027949

Dauksiene, A., Ruzauskas, M., Gruzauskas, R., Zavistanaviciute, P., Starkute, V., Lele, V., et al. (2021). A comparison study of the caecum microbial profiles, productivity and production quality of broiler chickens fed supplements based on medium chain fatty and organic acids. Animals 11:610. doi: 10.3390/ ani11030610

Deng, W., Dong, X. F., Tong, J. M., and Zhang, Q. (2012). The probiotic Bacillus licheniformis ameliorates heat stress-induced impairment of egg production, gut morphology, and intestinal mucosal immunity in laying hens. Poult. Sci. 91, 575-582. doi: 10.3382/ps.2010-01293

Diaz-Sanchez, S., D’Souza, D., Biswas, D., and Hanning, I. (2015). Botanical alternatives to antibiotics for use in organic poultry production. Poult. Sci. 94, 1419-1430. doi: 10.3382/ps/pev014

Dong, Y., Li, R., Liu, Y., Ma, L., Zha, J., Qiao, X., et al. (2020). Benefit of dietary supplementation with Bacillus subtilis BYS2 on growth performance, immune response, and disease resistance of broilers. Probiotics Antimicrob. Proteins 12, 1385-1397. doi: 10.1007/s12602-020-09643-w

Elokil, A. A., Magdy, M., Melak, S., Ishfaq, H., Bhuiyan, A., Cui, L., et al. (2020a). Faecal microbiome sequences in relation to the egg-laying performance of hens using amplicon-based metagenomic association analysis. Animal 14, 706-715. doi: 10.1017/S1751731119002428

Elokil, A. A., Abouelezz, K. F., Ahmad, H. I., Pan, Y., and Li, S. (2020b). Investigation of the impacts of antibiotic exposure on the diversity of the gut microbiota in chicks. Animals 10:896. doi: 10.3390/ani10050896

Frizzas de, L. A. C., Pizauro, J. M., Macari, M., and Malheiros, E. B. (2003). Effect of probiotic supplementation on performance and digestive enzymes activity of broiler chickens. Rev. Bras. De. Zootec. 32, 200-207.

Gadde, U., Kim, W. H., Oh, S. T., and Lillehoj, H. S. (2017). Alternatives to antibiotics formaximizing growth performance and feed efficiency in poultry: a review. Anim. Health Res. Rev. 18, 26-45. doi: 10.1017/S1466252316000207

Gao, Z., Wu, H., Shi, L., Zhang, X., Sheng, R., Yin, F., et al. (2017). Study of Bacillus subtilis on growth performance, nutrition metabolism and intestinal microflora of 1 to $42 \mathrm{~d}$ broiler chickens. Anim. Nutr. 3, 109-113. doi: 10.1016/j.aninu.2017. 02.002

Hadieva, G., Lutfullin, M., Pudova, D., Akosah, Y., Shagimardanova, E., Gogoleva, N., et al. (2021). Supplementation of Bacillus subtilis GM5 enhances broiler body weight gain and modulates cecal microbiota. 3 Biotech 11, 1-13. doi: 10.1007/s13205-020-02634-2

Hagerty, S. L., Hutchison, K. E., Lowry, C. A., and Bryan, A. D. (2020). An empirically derived method for measuring human gut microbiome alpha diversity: demonstrated utility in predicting health-related outcomes among a human clinical sample. PLoS One 15:e0229204. doi: 10.1371/journal.pone. 0229204

He, T., Long, S., Mahfuz, S., Wu, D., Wang, X., Wei, X., et al. (2019). Effects of probiotics as antibiotics substitutes on growth performance, serum biochemical parameters, intestinal morphology, and barrier function of broilers. Animals. 9:985. doi: 10.3390/ani9110985

Hong, Y., Cheng, Y., Li, Y., Li, X., Zhou, Z., Shi, D., et al. (2019). Preliminary study on the effect of Bacillus amyloliquefaciens TL on cecal bacterial community structure of broiler chickens. Bio. Med. Res. Int. 2019:5431354. doi: 10.1155/ 2019/5431354

Huang, Y., Li, Y., Huang, Q., Cui, Z., Yu, D., Rajput, I. R., et al. (2012). Effect of orally administered Enterococcus faecium EF1 on intestinal cytokines and chemokines productionof suckling piglets. Pak. Vet. J. 32, 81-84.

Jacquier, V., Nelson, A., Jlali, M., Rhayat, L., Brinch, K. S., and Devillard, E. (2019). Bacillus subtilis 29784 induces a shift in broiler gut microbiome toward butyrate-producing bacteria and improves intestinal histomorphology and animal performance. Poult. Sci. 98, 2548-2554. doi: 10.3382/ps/pey602
Jayaraman, S., Das, P. P., Saini, P. C., Roy, B., and Chatterjee, P. N. (2017). Use of Bacillus Subtilis PB6 as a potential antibiotic growth promoter replacement in improving performance of broiler birds. Poult. Sci. 96, 2614-2622. doi: 10.3382/ ps/pex079

Jumpertz, R., Le, D. S., Turnbaugh, P. J., Trinidad, C., Bogardus, C., Gordon, J. I., et al. (2011). Energy-balance studies reveal associations between gut microbes, caloric load, and nutrient absorption in humans. Am. Clin. J. Nutr. 94, 58-65. doi: 10.3945/ajcn.110.010132

Kareem, K. Y., Loh, T. C., Foo, H. L., Akit, H., and Samsudin, A. A. (2016). Effects of dietary postbiotic and inulin on growth performance, IGF1 and GHR mRNA expression, faecal microbiota and volatile fatty acids in broilers. BMC Vet. Res. 12:163. doi: 10.1186/s12917-016-0790-9

Lammers, A., Wieland, W. H., Kruijt, L., Jansma, A., Straetemans, T., Schots, A., et al. (2010). Successive immunoglobulin and cytokine expression in the small intestine of juvenile chicken. Dev. Comp. Immunol. 34, 1254-1262. doi: 10.1016/j.dci.2010.07.001

Lee, K. W., Lillehoj, H. S., Jang, S. I., and Lee, S. H. (2014). Effects of salinomycin and Bacillus subtilis on growth performance and immune responses in broiler chickens. Res. Vet. Sci. 97, 304-308. doi: 10.1016/j.rvsc.2014.07.021

Lee, K. W., Lillehoj, H. S., Jang, S. I., Li, G., Lee, S. H., Lillehoj, E. P., et al. (2010). Effect of Bacillus-based direct-fed microbials on Eimeria maxima infection in broiler chickens. Comp. Immunol. Microbiol. Infect. Dis. 33, e105-10. doi: 10.1016/j.cimid.2010.06.001

Li, C. L., Wang, J., Zhang, H. J., Wu, S. G., Hui, Q. R., Yang, C. B., et al. (2019). Intestinal morphologic and microbiota responses to dietary Bacillus spp. in a broiler chicken model. Front. Microbiol. 9:1968. doi: 10.3389/fphys.2018. 01968

Li, Y., Guo, B., Wu, Z., Wang, W., Li, C., Liu, G., et al. (2020). Effects of fermented soybean meal supplementation on the growth performance and cecal microbiota community of broiler chickens. Animals 10:1098. doi: 10.3390/ ani10061098

Liu, X., Peng, C., Qu, X., Guo, S., Chen, J. F., He, C., et al. (2019). Effects of Bacillus subtilis C-3102 on production, hatching performance, egg quality, serum antioxidant capacity and immune response of laying breeders. J. Anim. Physiol. Anim. Nutr. 103, 182-190. doi: 10.1111/jpn.13022

Ma, Y., Wang, W., Zhang, H., Wang, J., Zhang, W., Gao, J., et al. (2018). Supplemental Bacillus subtilis DSM 32315 manipulates intestinal structure and microbial composition in broiler chickens. Sci. Rep. 8:15358. doi: 10.1038/ s41598-018-33762-8

Nobutani, K., Sawada, D., Fujiwara, S., Kuwano, Y., Nishida, K., Nakayama, J., et al. (2017). The effects of administration of the Lactobacillus gasseri strain CP 2305 on quality of life, clinical symptoms and changes in gene expression in patients with irritable bowel syndrome. J. Appl. Microbiol. 122, 212-224. doi: $10.1111 /$ jam.13329

NRC (1994). Nutrient Requirements of Poultry: National Research Council. Washington: National Academy Press.

Palamidi, I., Fegeros, K., Mohnl, M., Abdelrahman, W. H. A., Schatzmayr, G., Theodoropoulos, G., et al. (2016). Probiotic form effects on growth performance, digestive function, and immune related biomarkers in broilers. Poult. Sci. 95, 1598-1608. doi: 10.3382/ps/pew052

Park, I., Lee, Y., Goo, D., Zimmerman, N. P., Smith, A. H., Rehberger, T., et al. (2020). The effects of dietary Bacillus subtilis supplementation, as an alternative to antibiotics, on growth performance, intestinal immunity, and epithelial barrier integrity in broiler chickens infected with Eimeria maxima. Poult. Sci. 99, 725-733. doi: 10.1016/j.psj.2019.12.002

Pedroso, A. A., Menten, J. F., Lambais, M. R., Racanicci, A. M. C., Longo, F. A., and Sorbara, J. O. B. (2006). Intestinal bacterial community and growth performance of chickens fed diets containing antibiotics. Poult. Sci. 85, 747-752. doi: $10.1093 / \mathrm{ps} / 85.4 .747$

Qin, C., Gong, L., Zhang, X., Wang, Y., Wang, Y., Wang, B., et al. (2018). Effect of Saccharomyces boulardii and Bacillus subtilis B10 on gut microbiota modulation in broilers. Anim. Nutr. 4, 358-366. doi: 10.1016/j.aninu.2018.03. 004

Rajput, I. R., Li, L. Y., Xin, X., Wu, B. B., Juan, Z. L., Cui, Z. W., et al. (2013). Effect of Saccharomyces boulardii and Bacillus subtilis B10 on intestinal ultrastructure modulation and mucosal immunity development mechanism in broiler chickens. Poult. Sci. 92, 956-965. doi: 10.3382/ps.2012-02845 
Rajput, I. R., and Li, W. F. (2012). Potential role of probiotics in mechanism of intestinal immunity. Pak. Vet. J. 32, 303-308.

Rajput, I. R., Ying, H., Yajing, S., Arain, M. A., Weifen, L., Ping, L., et al. (2017). Saccharomyces boulardii and Bacillus subtilis B10 modulate TLRs and cytokines expression patterns in jejunum and ileum of broilers. PLoS One 12:e0173917. doi: 10.1371/journal.pone.0173917

Riazi, S., Wirawan, R. E., Badmaev, V., and Chikindas, M. L. (2009). Characterization of lactosporin, a novel antimicrobial protein produced by Bacillus coagulans ATCC 7050. J. Appl. Microbiol. 106, 1370-1377. doi: 10.1111/ j.1365-2672.2008.04105.x

SAS (2002). Statistical Analysis System Proprietary Software. Version 9. Cary, NC: SAS Institute Inc.

Selvam, R., Maheswari, P., Kavitha, P., Ravichandran, M., Sas, B., and Ramchand, C. N. (2009). Effect of Bacillus subtilis PB6, a natural probiotic on colon mucosal inflammation and plasma cytokines levels in inflammatory bowel disease. Indian J. Biochem. Biophys. 46, 79-85.

Shaufi, M. A. M., Sieo, C. C., Chong, C. W., Gan, H. M., and Ho, Y. W. (2015). Deciphering chicken gut microbial dynamics based on high throughput 16S rRNA metagenomics analyses. Gut Pathog. 7:4. doi: 10.1186/s13099-0150051-7

Shokryazdan, P., Faseleh Jahromi, M., Liang, J. B., Ramasamy, K., Sieo, C. C., and Ho, Y. W. (2017). Effects of a Lactobacillus salivarius mixture on performance, intestinal health and serum lipids of broiler chickens. PLoS One 12:e175959.

Singer, R. S., Finch, R., Wegener, H. C., Bywater, R., Walters, J., and Lipsitch, M. (2003). Antibiotic resistance the interplay between antibiotic use in animals and human beings. Lancet. Infect. Dis. 3, 47-51. doi: 10.1016/s1473-3099(03) 00490-0

Sun, Y. Z., Yang, H. L., Ma, R. L., Zhang, C. X., and Lin, W. Y. (2011). Effect of dietary administration of Psychrobacter sp. on the growth, feed utilization, digestive enzymes and immune responses of grouper Epinephelus coioides. Aquac. Nutr. 17, e733-e740. doi: 10.1111/j.1365-2095.2010.00837.x

Suresh, G., Das, R. K., Kaur Brar, S., Rouissi, T., Avalos Ramirez, A., Chorfi, Y., et al. (2018). Alternatives to antibiotics in poultry feed: molecular perspectives. Crit. Rev. Microbiol. 44, 318-335. doi: 10.1080/1040841X.2017.1373062

Tang, K. L., Caffrey, N. P., Nóbrega, D. B., Cork, S. C., Ronksley, P. E., Barkema, H. W., et al. (2017). Restricting the use of antibiotics in food-producing animals and its associations with antibiotic resistance in food-producing animals and human beings: a systematic review and meta-analysis. Lancet. Planet Health 1, e316-e327. doi: 10.1016/S2542-5196(17)30141-9

Trela, J., Kierończyk, B., Hautekiet, V., and Józefiak, D. (2020). Combination of Bacillus licheniformis and salinomycin: effect on the growth performance and git microbial populations of broiler chickens. Animals 10:889. doi: 10.3390/ ani10050889

Wang, H., Ni, X., Qing, X., Liu, L., Lai, J., Khalique, A., et al. (2017). Probiotic enhanced intestinal immunity in broilers against subclinical necrotic enteritis. Front. Immunol. 8:1592. doi: 10.3389/fimmu.2017.01592

Wang, Y. H., Xu, M., Wang, F. N., Yu, Z. P., Yao, J. H., Zan, L. S., et al. (2009). Effect of dietary starch on rumen and small intestine morphology and digesta $\mathrm{pH}$ in goats. Livest. Sci. 122, 48-52. doi: 10.1016/j.livsci.2008.07.024
Wei, S., Morrison, M., and Yu, Z. (2013). Bacterial census of poultry intestinal microbiome. Poult. Sci. 92, 671-683. doi: 10.3382/ps.201202822

Xiao, Y., Xiang, Y., Zhou, W., Chen, J., Li, K., and Yang, H. (2017). Microbial community mapping in intestinal tract of broiler chicken. Poult. Sci. 96, 13871393. doi: $10.3382 / \mathrm{ps} /$ pew372

Yang, Y., Ashworth, A. J., Willett, C., Cook, K., Upadhyay, A., Owens, P. R., et al. (2019). Review of antibiotic resistance, ecology, dissemination, and mitigation in US broiler poultry systems. Front. Microbiol. 10:2639. doi: 10.3389/fmicb. 2019.02639

Zhang, L., Bai, K., Zhang, J., Xu, W., Huang, Q., and Wang, T. (2017). Dietary effects of Bacillus subtilis fmbj on the antioxidant capacity of broilers at an early age. Poult. Sci. 96, 3564-3573. doi: 10.3382/ps/pex172

Zhang, L., Zhang, L., Zeng, X., Zhou, L., Cao, G., and Yang, C. (2016). Effects of dietary supplementation of probiotic, Clostridium butyricum, on growth performance, immune response, intestinal barrier function, and digestive enzyme activity in broiler chickens challenged with Escherichia coli K88. J. Anim. Sci. Biotechnol. 7:3. doi: 10.1186/s40104-0160061-4

Zhang, Z. F., Zhou, T. X., Ao, X., and Kim, I. H. (2012). Effects of $\beta$-glucan and Bacillus subtilis on growth performance, blood profiles, relative organ weight and meat quality in broilers fed maize-soybean meal based diets. Livest. Sci. 150, 419-424. doi: 10.1016/j.livsci.2012.10.003

Zheng, Z., Ma, C., Gao, C., Li, F., Qin, J., Zhang, H., et al. (2011). Efficient conversion of phenylpyruvic acid to phenyllactic acid by using whole cells of Bacillus coagulans SDM. PLoS One 6:e19030. doi: 10.1371/journal.pone. 0019030

Zhu, X., Liu, J., Liu, H., and Yang, G. (2020). Soybean oligosaccharide, stachyose, and raffinose in broilers diets: effects on odor compound concentration and microbiota in cecal digesta. Poult. Sci. 99, 3532-3539. doi: 10.1016/j.psj.2020. 03.034

Conflict of Interest: The authors declare that the research was conducted in the absence of any commercial or financial relationships that could be construed as a potential conflict of interest.

Publisher's Note: All claims expressed in this article are solely those of the authors and do not necessarily represent those of their affiliated organizations, or those of the publisher, the editors and the reviewers. Any product that may be evaluated in this article, or claim that may be made by its manufacturer, is not guaranteed or endorsed by the publisher.

Copyright (C) 2022 Mohamed, Sun, Bumbie, Elokil, Mohammed, Zebin, Hu, Wu and Tang. This is an open-access article distributed under the terms of the Creative Commons Attribution License (CC BY). The use, distribution or reproduction in other forums is permitted, provided the original author(s) and the copyright owner(s) are credited and that the original publication in this journal is cited, in accordance with accepted academic practice. No use, distribution or reproduction is permitted which does not comply with these terms. 